\title{
Introdução ao uso dos protocolos SRU/SRW: ferramentas para a catalogação cooperativa
}

Walter Moreira

\author{
Doutorando em Ciência da Informação \\ (ECA/USP).Professor das Faculdades \\ Integradas Teresa D'Ávila (Fatea).
}

Thiago Ribeiro

\author{
Especialista em Administração: \\ Tecnologia da Informação (Fatea) \\ Gerente de Tecnologia Flying Fishes - \\ Cineclick.
}

Apresenta e discute o uso dos protocolos Search and Retrieve URL (SRU) / Search/Retrieve Web Service (SRW), ambos desenvolvidos e mantidos pela Biblioteca do Congresso nos Estados Unidos. O SRU/SRW é uma nova proposta de protocolo baseada no já disseminado protocolo Z39.50, cujo fim é facilitar o desenvolvimento de soluções para ações de catalogação cooperativa de informações bibliográficas. Apresenta alguns exemplos de aplicação dos protocolos e identifica a ausência de produção sobre o assunto em periódicos especializados.

Palavras-chave: SRU; SRW; Z39.50; Protocolos de comunicação; catalogação cooperativa.

\section{Introduction to the use of protocols SRU/SRW: tools for cooperative cataloging}

In this article the use of Search and Retrieve URL (SRU) / Search and Retrieve Web Service (SRW) protocols, both developed and maintained by the Library of Congress in the United States, is presented and discussed. The $S R U / S R W$ is a new draft protocol based on the already widespread Z39.50 protocol, whose purpose is to facilitate the development of solutions for shared cooperative cataloging of bibliographic information. Some examples of 
applications are also shown and the lack of production on the subject in specialized journals is pointed out.

Keywords: SRU; SRW; Z39.50; Communication protocols; Cooperative cataloging.

\section{Introdução}

O crescimento do volume de informações disponíveis atualmente, o qual não se cansa de adjetivar de vertiginoso, traz como imperativo a cooperação entre as diversas unidades de informação (compreendidas aqui as bibliotecas e todas as outras). Não se trata, formalmente, de mudança significativa no fazer biblioteconômico, mas de aceleração do processo, tendo em vista, principalmente, as facilidades engendradas pelas tecnologias da informação e pelos avanços da telemática.

Chama-se à responsabilidade a Ciência da Informação e a Biblioteconomia, ciências socialmente aplicadas, é preciso enfatizar, cujos conjuntos de conhecimento teórico ou prático situam-se perfeitamente neste cenário. A Ciência da Informação preocupa-se essencialmente com o fluxo que envolve a comunicação do conhecimento e seus registros, compreendidos aqui a produção, a circulação e o uso. Saracevic (1996, p.47) a define como

um campo dedicado às questões científicas e à prática profissional voltadas para os problemas de efetiva comunicação do conhecimento e de seus registros entre os seres humanos, no contexto social, institucional ou individual do uso e das necessidades de informação. No tratamento destas questões são consideradas de particular interesse as vantagens das modernas tecnologias informacionais.

A preocupação demonstrada pelo autor com as "modernas tecnologias informacionais" confere um caráter indispensável às definições clássicas: a peremptoriedade. Julgar como sendo de interesse particular as tecnologias torna o objeto da Ciência da Informação constantemente renovado, pois implica em rever continuamente o seu modo de proceder na prática. A Ciência da Informação responsabiliza-se socialmente, portanto, pelas soluções mediadoras entre a explosão documental e a diversidade de ofertas e critérios de busca e recuperação de informações registradas.

As bibliotecas vêm, tradicionalmente, catalogando recursos informacionais e empreendendo esforços em prol da cooperação e da padronização, entendendo-se esta como um elemento condicionador daquela. A catalogação de informação na publicação e o sucesso do formato MARC podem ser apontados como exemplos. 
A produção de catálogos visa a prover identificadores para um nível imediato de descrição das características físicas e intelectuais do documento (MARCUM, 2005). Marcum levanta algumas questões interessantes e provocadoras em relação à catalogação atualmente, dentre elas: a) Tendo-se em vista que há diversos livros e periódicos acessíveis online, deve-se considerar os mecanismos de busca como meio principal de localização e acesso? b) Com o MARC generalizado e espalhado pelo mundo, como promover mudanças sem criar o caos? São questões que evidenciam a atualidade da problemática. Em outras palavras, é preciso discutir qual é exatamente o papel reservado aos metadados e aos catálogos de bibliotecas neste cenário.

Fast e Campbell (2004) compararam o nível de percepção e de interação que os universitários possuem em relação aos catálogos de acesso público online (OPACs) com os níveis de percepção e interação que experimentam em relação ao Google, e identificaram preferência por este último. Uma das principais razões apontadas para a preferência foi a facilidade de uso. Os autores sugerem, com base nos resultados, que o design dos atuais OPACs sejam agressivamente redefinidos em face dos padrões de usabilidade da web. O Google tem sido celebrado pela eficácia de seu algoritmo de busca, mas num nível imediato de aproximação é sua interface limpa e eficaz que seduz o usuário médio, aquele usuário sem conhecimentos avançados de informática ou de estratégias de busca de informação.

Não se pode negar que os hábitos adquiridos com a navegação na internet influenciam o modo como o usuário médio busca informação em bibliotecas. Acredita-se, entretanto, que o usuário universitário, ou outro de mesmo nível, ainda prefira a biblioteca pelo que tem de confiabilidade. Seus catálogos colocam o usuário em contato com fontes de informação que foram, de uma ou de várias formas, avaliadas. Marcum (2005) provoca novamente: é possível repensar a catalogação para encontrar algo semelhante ao mundo Google? Sem ações de cooperação e de harmonização em nível de catalogação e busca, sequer um patamar próximo poderá ser alcançado. A simplicidade aliada à eficácia garantida por acordos de cooperação e de padronização, conforme indicado acima, parecem ser o tom da mudança.

O acordo é uma das variáveis condicionantes de sucesso para as ações conjuntas. Por essa razão, objetiva-se discutir neste artigo alguns dos protocolos utilizados para comunicação e troca de informações bibliográficas entre bibliotecas.

Um protocolo, em sentido corriqueiro, refere-se ao estabelecimento de acordos. É assim que se tem falado muito ultimamente do Protocolo de Kioto, por exemplo. Menos importante do que um tratado ou uma convenção, um protocolo visa a dar garantias de comunicação entre as nações envolvidas na questão. Se no mundo dos homens é assim, mais necessário ainda são os protocolos no mundo das máquinas. Em informática, um protocolo é entendido como o conjunto de sinais, códigos e regras pré-combinadas para serem usadas na troca de dados entre sistemas dispostos em rede. Aliás, quando se fala em redes de 
informação, fala-se também, necessariamente, em uma "sopa de letrinhas" referentes a protocolos, algumas já famosas e utilizadas na linguagem do dia-a-dia, como o HTTP (Hypertext Transfer Protocol) e o URL (Unified Resource Locator), por exemplo; outros ainda relativamente desconhecidos, mesmo entre a pequena parcela de seus usuários potenciais, como é o caso do Search/Retieve URL (SRU) e do Search/Retrieve Web Services (SRW), discutidos aqui em face de seu relativo desconhecimento pela comunidade bibliotecária.

Discutindo, portanto, de forma geral, os protocolos de comunicação, objetiva-se especificamente, com o presente artigo, apresentar e discutir o protocolo SRU/SRW e suas facilidades, melhorias e avanço em relação ao protocolo Z39.50, o qual foi reescrito para se tornar mais fácil e acessível aos profissionais que trabalham na área da organização da informação, tendo em vista igualmente os profissionais das áreas de Ciência da Computação e Engenharia de Software, que desenvolvem aplicativos para unidades de informação e que necessitam, do mesmo modo, recuperar dados com facilidade.

\section{Revisitando o Z39.50}

O Z39.50 antecede a popularidade da $w e b$, tendo surgido em 1970 (CABRAL, 2007) e se consolidado nos anos 80 . Foi muito bem recebido como solução para um dos mais velhos problemas da catalogação bibliotecária: o re-trabalho, a duplicação desnecessária na produção de informações documentárias em nível descritivo. A idéia de criar uma interface que poderia permitir, numa situação ideal, a catalogação única de um documento era realmente promissora, num cenário em que já era presente uma larga preocupação com o volume crescente de documentos. A informática já havia avançado de forma inexorável e já se experimentava, mesmo no Brasil, o surgimento de veículos alternativos ao texto científico, diferençados do tradicional códex impresso.

Desenvolvido pela Library of Congress, o Z39.50 é um protocolo de comunicação entre computadores distribuídos em rede com a finalidade de viabilizar a pesquisa, a recuperação e o intercâmbio de informações bibliográficas. Baseia-se numa arquitetura cliente/servidor e possibilita o uso do formato MARC para a implementação de ações de catalogação cooperativa.

Apesar da idade, o Z39.50 ainda é relativamente desconhecido fora do ambiente stricto da Biblioteconomia. Geralmente tratado como uma questão fundamentalmente técnica da própria área, o protocolo é pouco difundido no mundo da Ciência da Computação e, conseqüentemente, é, considerando-se sua importância, subutilizado nas mais diversas aplicações de sistemas de informação bibliográficos ou documentários. Por outro lado, quando conhecido pelo desenvolvedor, este protocolo também Ihe impõe um conhecimento mais elaborado das técnicas biblioteconômicas para utilizá-lo, o que prejudica a eficácia da recuperação da informação nas aplicações desse gênero quando não há desenvolvimento conjunto entre bibliotecário e desenvolvedor. Os 
prejuízos mais destacados neste descompasso são a dificuldade de localização e recuperação de informações bibliográficas que se sabem já existentes, e o já mencionado re-trabalho.

\section{SRU / SRW: a nova geração}

O projeto de reestruturação do protocolo $Z 39.50$ foi inicialmente denominado pelo acrônimo ZIG/ZING (Z39.50 next generation), posteriormente modificado para SRU. Na esteira do Z39.50, utilizado mais especificamente pelas bibliotecas chamadas tradicionais, surgiram os protocolos desenhados para aplicações digitais, o SRU e, posteriormente, - SRW, ambos desenvolvidos pela Library of Congress, nos Estados Unidos. Os protocolos SRU/SRW trouxeram as facilidades do Z39.50 para o contexto da internet, em ambientes de URL e de serviços web.

A introdução do SRU mudou amplamente o cenário; a própria abrangência da nova proposta torna-se sua aliada mais eficaz para a realização do que propõe. Por tratar-se de um protocolo padrão mensurado através do protocolo HTTP, protocolo para a navegabilidade de sites na internet, pode ser utilizado largamente pelos desenvolvedores de software.

Com o SRU, um leque muito grande de possibilidades se abre quanto à harmonização na catalogação de dados. Isto é possível porque o protocolo SRU recupera registros no formato MARC, utilizado para catalogar registros de bibliotecas, e Dublin Core, utilizado para catalogar arquivos digitais, os quais estão entre os principais formatos utilizados na catalogação de informações documentárias.

\section{SRU}

O SRU utiliza o tipo de serviço Web REST-ful (Representational State Transfer). Este serviço codifica comandos do cliente para o servidor em uma string, ou cadeia de caracteres, na forma de um URL. Cada um desses valores é especificado no formato nome=valor e a cada nova especificação é atribuído um novo parâmetro para o servidor (FIG. 1). 


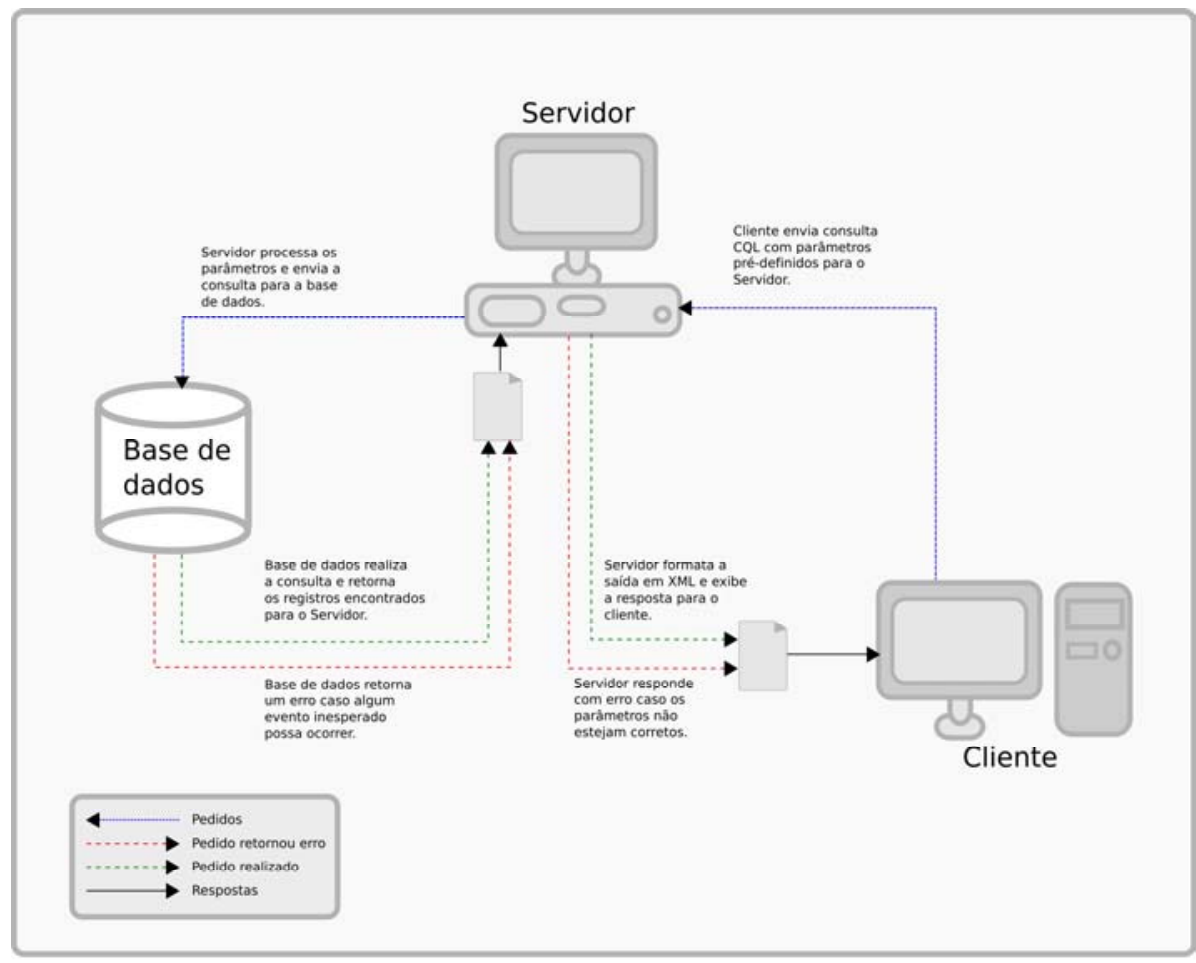

Figura 1. Fuuxo de intormachăo no SRL

FIGURA 1 - Fluxo de informação no SRU.

Fonte: Elaborado pelos autores.

O servidor processa estes parâmetros pré-estabelecidos e retorna os valores no formato eXtensible Markup Language (XML). O XML foi estabelecido pela World Wide Web Consortium (W3C) como um padrão para construir marcações de hipertexto. Siqueira (2003) aborda este assunto de maneira extensa e aprofundada em sua dissertação de mestrado, mostrando as várias vantagens da marcação utilizando o formato $X M L$, nas áreas de domínio da Ciência da Informação.

É importante lembrar que os parâmetros são sensitive-case, ou seja, devem ser escritos da maneira em que são apresentados, não podendo haver diferenciação na maneira em que são escritos. No QUADRO 1 apresenta-se um exemplo de estrutura de URL SRU:

\section{QUADRO 1 - Estrutura de URL SRU}

Protocolo://EnderecoDoServidor:Porta/NomeDaBaseDeDados?Versao=Numero DaVersao\&Operacao $=$ TipoDaOperacao $\&$ Consulta $=$ Consulta $\&$ OpcoesRetorno $=$ Opcoes\&ParametrosRequisicao $=$ Parametros

Aplicando-se o exemplo a uma situação real de busca pelo termo dinossauro no servidor da Biblioteca do Congresso dos Estados Unidos (QUADRO 2): 
QUADRO 2 - URL SRU de uma busca por "dinossauro" na LOC

http://z3950.loc.gov:7090/voyager?version=1.1\&operation=searchRetrieve\&query=dinosaur \&maximumRecords $=1 \&$ recordSchema $=\mathrm{dc}$

Nas linhas abaixo o SRU será desmembrado visando à sua melhor compreensão:

- protocolo: http;

- endereço do servidor: z3950.loc.gov;

- porta: 7090 (este item não é obrigatório, podendo variar de acordo com a configuração de cada servidor);

- nome da base de dados: voyager;

versão: refere-se à versão do protocolo. No exemplo apresentado, é utilizada a versão 1.1. Quando houver identidade de versão entre cliente e servidor, a requisição dar-se-á de modo normal. Caso cliente e servidor utilizem versões diferentes, poderão acontecer os seguintes casos:

- se o cliente fizer um pedido de versão 2.0 em um servidor 1.1, a resposta segue a versão mais baixa e

- se o cliente fizer um pedido de versão $1.1 \mathrm{em}$ um servidor 2.0, o servidor irá rebaixar a resposta para o pedido do cliente.

operação: existem três tipos de operações que podem ser utilizadas no serviço SRU/SRW, são elas: explain, scan e searchRetrieve:

- explain: utilizada pelo cliente para obter mais informações sobre a situação do servidor. Algumas opções que são listadas pelo explain são: versão, bases de dados, localização das bases, serviços oferecidos etc;

- scan: utilizada para listar e numerar os termos encontrados em uma pesquisa na base de dados. Deve ser utilizada com a opção scanClause para se fazer a busca do termo desejado. A busca pelo termos mundo, por exemplo, na base voyager, seria representada assim: http://z3950.loc.gov: $7090 /$ voyager?operation=scan\&scanClau $\underline{\text { se }=\text { mundo }}$

searchRetrive: a operação principal do serviço SRU/SRW. Identifica a requisição solicitada, realiza a busca na base de dados e retorna os resultados encontrados. Os resultados podem ser requeridos de acordo com a definição do cliente. Estes resultados podem ser retornados em MARCXML, Dublin Core, MODS (Metadata Object Description Schema) e outras opções. Caso nenhum parâmetro seja definido para a saída dos dados, o servidor utilizará o padrão de saída DC (Dublin Core). Esta opção deve ser utilizada com o parâmetro query para se fazer a busca do termo desejado. Seguindo-se com o exemplo de busca pelo termo mundo, temse:

http://z3950.loc.gov:7090/voyager?operation=searchRetrieve\&query=mu ndo; 
- consulta: as consultas são expressas em Common Query Language (CQL) para versão 1.1 e para novas versões como Contextual Query Language (CQL), uma linguagem formal utilizada para representar pesquisas em sistemas de informação. Como mostrado anteriormente, utiliza-se o parâmetro scanClause para a operação Scan e o parâmetro query para searchRetrive. O QUADRO 3 apresenta exemplos de como as consultas podem construídas utilizando-se CQL.

$\bullet$

QUADRO 3- Exemplos de consultas em CQL

\begin{tabular}{r|l}
\hline title all "Síntese fala" & Título contém todas as palavras entre aspas \\
\hline title any "Syntactical annotation" & Título contém qualquer uma das palavras entre aspas \\
\hline title exact "The Multilingual Question \\
Answering Track at CLEF" & Título exato \\
\hline date within “2002 2006" & Datas entre 2002 e 2006 \\
\hline any/relevant "Syntactical annotation" & $\begin{array}{l}\text { Aplica um algoritmo de relevância para determinar } \\
\text { resultados e a ordem respectiva }\end{array}$ \\
\hline
\end{tabular}

Fonte: CABRAL (2007, p. 38).

Opções mais detalhadas, como operações booleanas e outros operadores, podem ser encontradas em http://www.loc.gov/standards/sru/specs/cql.html ${ }^{1}$.

opções de retorno: podem ser inseridas como parâmetros para filtrar ou alterar a exibição dos resultados encontrados. O QUADRO 4, na próxima página, apresenta algumas destas opções que podem ser combinadas para trazerem o resultado pretendido.

QUADRO 4- Exemplos de opções de retorno

\begin{tabular}{r|l}
\hline numberOfRecords & $\begin{array}{l}\text { Determina a quantidade de registros que devem ser retornados. } \\
\text { Caso a operação tenha falhado, o resultado será } 0 .\end{array}$ \\
\hline resultSetIdleTime & $\begin{array}{l}\text { O tempo de segundos em que a consulta ainda ficará disponível. } \\
\text { Após este tempo a consulta será expirada. }\end{array}$ \\
\hline extraResponseData & Informações extras fornecidas pelo servidor. \\
\hline records & $\begin{array}{l}\text { Se algum problema ocorrer, o servidor poderá retornar o motivo. } \\
\text { Os diagnósticos podem ser classificados como fatal e non-fatal. }\end{array}$ \\
\hline
\end{tabular}

Fonte: Elaborado pelos autores.

\footnotetext{
${ }^{1}$ Acesso em: 10 ago. 2007.
} 


\section{SRW}

Com os mesmos propósitos do SRU, foi desenvolvido o SRW, sendo que este se diferencia daquele pelo uso de um serviço pré-estabelecido, o Simple Object Access Protocol (SOAP), e não de um URL.

O SOAP presta-se à encapsular e transportar as chamadas de procedimento remoto (Remote Procedure Calls, ou RPCs), criando mensagens estruturadas no formato XML para a troca de informação em ambientes remotos. Definido de modo mais simples, pode-se compreendê-lo como um protocolo para acessar um serviço web e trazer interatividade para a aplicação (W3C, 2007).

$O$ uso do SOAP tem como vantagem a sua independência em relação às linguagens de programação, sua simplicidade e o fato de ser extensível, o que permite sua utilização em qualquer aplicação. Pode-se citar ainda como vantagem o fato do SOAP poder realizar suas chamadas sobre o protocolo HTTP e ser estruturado em XML, ou seja, duas tecnologias instituídas como padrão e usadas em larga escala.

Conectando-se através do protocolo http, diminui-se a possibilidade de que qualquer firewall que esteja entre o servidor e o cliente possa vir a bloquear estas chamadas. Muitas aplicações fazem RPCs através de portas específicas, ou pré-determinadas pela própria aplicação, mas, se estas portas não estiverem liberadas no firewall, a aplicação não logrará sucesso em sua execução (FIG. 2).

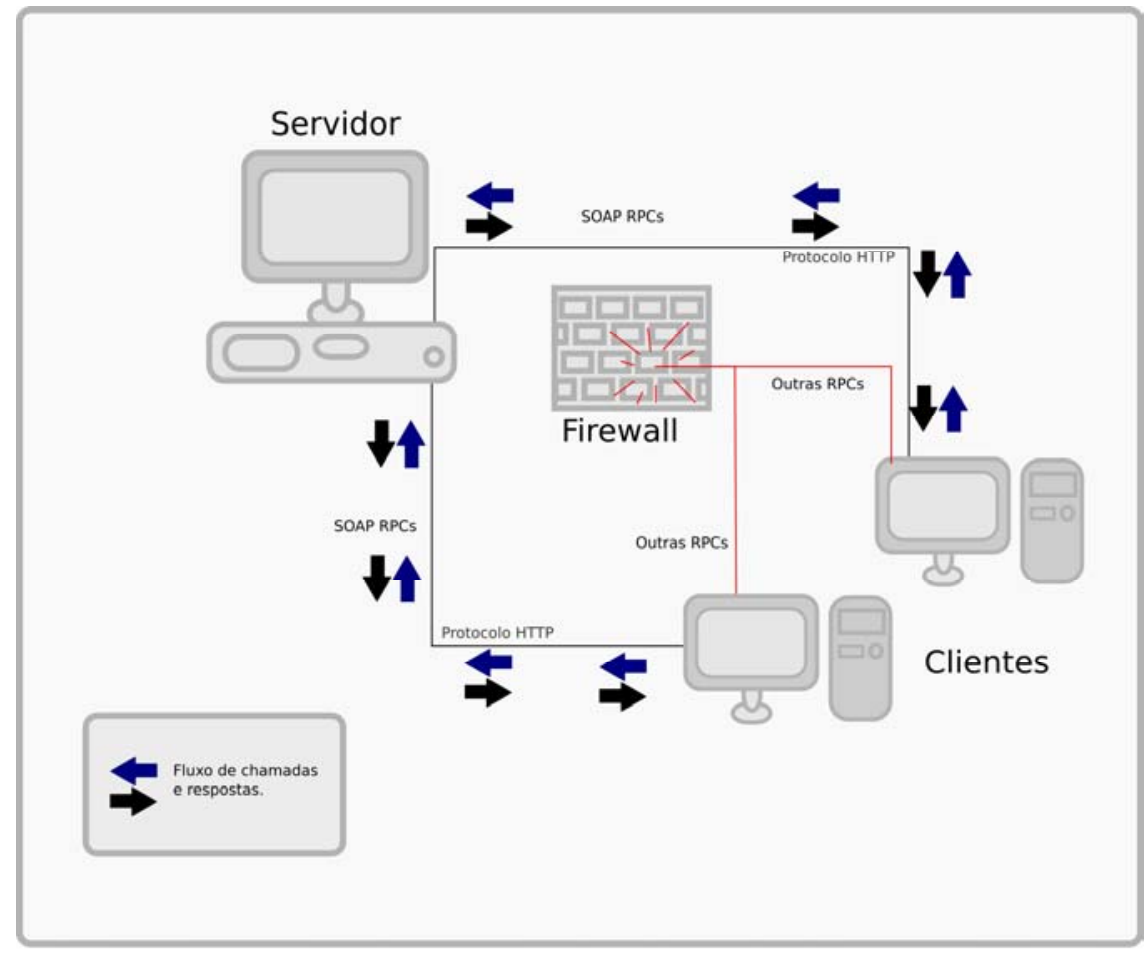

Figura 2 - Fluxo com mensagem SOAP em rede com Firewal

FIGURA 2 - Fluxo com mensagem SOAP em rede com firewall. Fonte: Elaborado pelos autores. 
Várias linguagens de desenvolvimento possuem bibliotecas, funções e métodos próprios para a facilitação do uso do protocolo SOAP, tais como: PHP, Java, ASP, Python, $\mathrm{C}++$, etc. Para se construir uma noção da representatividade dessas linguagens, é possível consultar o seu índice de uso medido pela Tiobe Software (2007), cujo método consiste na contagem de hits nos mecanismos de busca mais populares em estratégias de busca combinadas ${ }^{2}$.

Uma mensagem SOAP é composta por três elementos: envelope, cabeçalho e corpo (Figura 3). O envelope, por conter o cabeçalho e o corpo, é o elemento principal de uma mensagem SOAP, sem o qual fica impossível a utilização do protocolo. O cabeçalho, ou header, é um elemento opcional no envelope, e seu uso se aplica quando a mensagem deve ser enviada para um determinado nó (node). Caso seja necessária sua utilização, esta deverá ser a primeira informação no envelope. O corpo ou body é um elemento obrigatório no envelope; afinal, sem um conteúdo definido não faz sentido algum enviar ou receber qualquer tipo de mensagem. Dentro do corpo encontra-se o payload, que é a informação que se quer transportar para o destino final. O corpo também pode transmitir e receber mensagens de erro e alerta através do recurso opcional fault, próprio para estes fins.

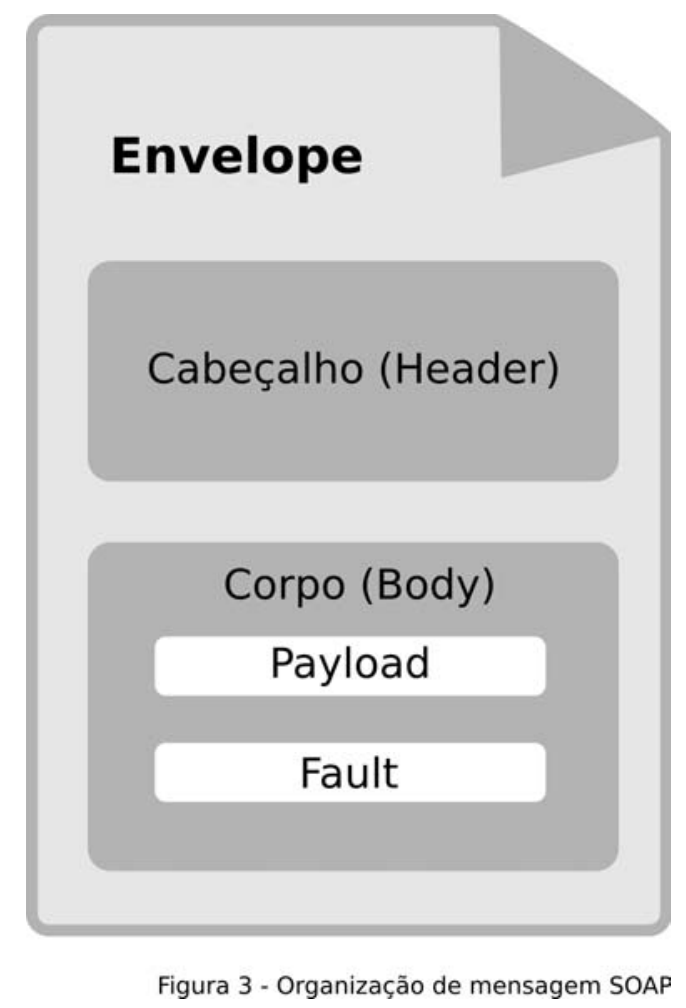

FIGURA 3 - Organização de mensagem em SOAP.

Fonte: Elaborado pelos autores.

\footnotetext{
${ }^{2}$ Disponível em: <http://www.tiobe.com/tpci.htm>. Acesso em: 28 abr. 2008.
} 
Tendo-se em vista que se discutem aqui elementos que possam facilitar a localização e a recuperação de informação em redes de informação, o SOAP torna-se uma alternativa muito interessante. Como não se trata, normalmente, de dados confidenciais, pode-se usufruir desta ferramenta sem preocupações.

Estes protocolos de busca e recuperação baseadas em XML são semanticamente compatíveis com o Z39.50, pois adaptam a maioria das suas partes ao ambiente web. Isso possibilita aos usuários o fornecimento de portas SRU/W para as aplicações Z39.50 existentes. O SRW pode ser combinado, por exemplo, com o Open Archives Initiative Protocol for Metadata Harvesting (OAI-PMH), no intuito de oferecer ferramentas poderosas e flexíveis para a recuperação de recursos na internet (MCCALLUM, 2004).

Como se pode perceber, a diferença fundamental entre os protocolos SRU e SRW é formal, reside na forma como a solicitação é realizada. A simplicidade do SRU em comparação com a necessidade de geração de um objeto $X M L$, pelo SRW, entretanto, tem lhe dado vantagem no uso (CABRAL, 2007, p. 38; BASSI, 2005).

\section{Alguns exemplos de uso do SRU/SRW}

Machado e Borbinha (2007) descrevem a aplicação do SRU em intranets, apresentando o sistema que denominam Mitra, "uma solução para indexação de conteúdos em linha e metadados descritivos complementares codificados em qualquer esquema XML", utilizando o Dublin Core como esquema de representação de metadados e fornecimento de interface SRU/W. O sistema promete uma combinação no mínimo merecedora de um olhar mais cuidadoso: nas palavras dos autores, "o poder da indexação livre de conteúdos com o poder do processamento de metadados estruturados, oferecendo o melhor dos dois mundos".

Embora desenhado como solução para intranet, o Mitra, esclarecem os autores, "pode ser totalmente administrado remotamente por outros sistemas como é o caso do serviço DiTeD, o repositório de Teses e Dissertações Digitais da Biblioteca Nacional Digital, que o usa como motor de busca interno para indexar as suas coleções". A aplicação pode ser conferida em http://dited.bn.pt/ ${ }^{3}$.

Os protocolos de comunicação têm recebido, de modo geral, pouca atenção na literatura especializada da Ciência da Informação. Um levantamento ${ }^{4}$ realizado em periódicos brasileiros da área de Biblioteconomia/Ciência da Informação disponíveis eletronicamente (Ciência da Informação, DataGramaZero, Em Questão, Informação \& Informação, Informação \& Sociedade: estudos, Perspectivas em Ciência da Informação, Revista ACB, Revista Brasileira de Biblioteconomia e Documentação, Revista Digital de Biblioteconomia e Ciência da

\footnotetext{
${ }^{3}$ Acesso em: 28 abr. 2008.

${ }^{4}$ Realizado em 16 jul. 2007. Quando disponível utilizou-se o próprio mecanismo de busca do periódico, quando não, utilizaram-se recursos de busca avançada via Google.
} 
Informação e Transinformação) mostra a baixa ocorrência de textos sobre os protocolos aqui mencionados. O período de cobertura do levantamento refere-se ao período de disponibilidade na internet do periódico. Este levantamento foi realizado em 16 de julho de 2007. Quando disponível, utilizou-se o próprio mecanismo de busca do periódico, quando não, utilizaram-se recursos de busca avançada do Google.

Localizou-se apenas um artigo contemplando especificamente 0 protocolo Z39.50, o breve artigo de Rosetto (1997). Os protocolos SRU/W não são sequer mencionados. Trata-se claramente de um descompasso quando na mesma literatura grassam os trabalhos sobre perspectivas de registro e recuperação de informações em rede e sobre sociedades denominadas da informação ou do conhecimento. Chumbe, MacLeod e Kennedy (2007) identificaram a mesma ausência na literatura de língua inglesa. Guardadas as devidas proporções, os autores identificaram que o Open Archives Initiative Protocol for Metadata Harvesting (OAI-PMH) tem recebido maior atenção quando se trata de interoperabilidade.

\section{Considerações finais}

As bibliotecas confundem-se com os sistemas de informação ou com os sistemas de recuperação da informação, entre outras denominações, a partir do desenvolvimento das tecnologias aplicadas à informação, quando os processos de tratamento e recuperação da informação são automatizados e a perspectiva sistêmica pode ser instaurada com maior força e mais comodidade no trabalho bibliotecário. O que alavanca e sustenta os processos de cooperação em rede, contudo, são as tecnologias da informação, pois a prática é anterior.

Verificou-se no Pós-guerra, principalmente a partir dos estudos de Bush (1945), a necessidade do desenvolvimento de interfaces individualizadas, as quais poderiam possibilitar a oferta de caminhos particularizados de acesso ao espaço informacional. São dessa época, por exemplo, os índices de concordância (como o KWIC), tecnologia já ultrapassada mas ainda utilizada. A evolução verificada na década de sessenta estabelece conceitos como os de revocação e precisão, além das primeiras questões sobre a utilização de linguagem natural nas negociações (proposição e resposta) de busca.

A década de setenta vê surgir os sistemas de grande aplicação e o nascimento da noção de trabalho cooperativo na catalogação, utilizando ainda sistemas relativamente simples e limitados. $O$ constante crescimento da tecnologia em processamento de texto e o equivalente decréscimo no custo dos dispositivos de armazenamento e equipamentos de informática em geral, verificados principalmente a partir dos anos oitenta, significaram mais informação disponível em formato legível por máquina, tornando comum o fluxo da informação eletrônica. São dessa década os OPACs e as pesquisas em texto completo.

Os processos de catalogação representam para as bibliotecas, neste cenário, o imperativo da cooperação como condição de evitar o retrabalho e a imprecisão nos registros. A perspectiva da cooperação 
viabilizada pelo uso de padrões de catalogação e protocolos de comunicação, como os discutidos aqui, descentraliza e amplifica a conferência dos registros, tornando-os mais confiáveis.

Comparando-se com o Z39.50, numa perspectiva ainda subjetiva que depende de validação experimental, os protocolos SRU/W apresentam mais facilidade de implementação e uso, e são, por essas razões, mais acessíveis aos profissionais da informação em sentido lato, tanto àqueles mais ligados às práticas de organização da informação quanto àqueles que instrumentalizam a área desenvolvendo aplicativos. Utilizando-se, portanto, o SRU/W é possível estender a aplicabilidade dos padrões de catalogação, gerar metadados mais consistentes e diminuir a margem de erro causada pelo fator humano em catalogação de dados, trazendo mais consistência ao sistema e mais benefícios para o usuário da informação.

\section{Referências}

BASSI, M. C. Formati MARC-XML e servizi derivati: resoconto sulla sessione al congresso IFLA 2005. Bibliotime, v. 8, n. 3, p. 1-6, 2005. Disponível em: <http://didattica.spbo.unibo.it/bibliotime/num-viii3/bassi.htm >. Acesso em: 13 jul. 2007.

BUSH, V. As we think. The Atlantic Montly, v. 176, n. 1, p. 101-108, 1945. Disponível em: <www.theatlantic.com/doc/194507/bush>. Acesso em: 28 abr. 2008.

CABRAL, L. M. SUPeRB: Sistema Uniformizado de Pesquisa de Referências Bibliográficas. 2007. 130 f. Dissertação (Mestrado em Engenharia Informática) - Faculdade de Engenharia, Universidade do Porto, Porto, $2007 . \quad$ Disponível em: <http://acdc.linguateca.pt/LuisCabral/publicacoes/Proposta SUPERB.pdf> . Acesso em: 16 jul. 2007.

CHUMBE, S.; MaCLEOD, R.; KENNEDY, M. L. Building bridges with blocks: assisting digital library and virtual learning environment integration through reusable middleware. In: INTERNATIONAL CONFERENCE ON ELECTRONIC PUBLISHING: AWARENESS DISCOVERY AND ACCESS, 11., 2007, Vienna. Proceedings... Vienna, 2007. Disponível em: <http://eprints.rclis.org/archive/00009545/>. Acesso em: 16 jul. 2007.

FAST, K. V.; CAMPBELL, G. D. "I still like Google": university student perceptions of searching OPACs and the web. Proceedings of the American Society for Information Science and Technology, v. 41, n. 1, p. 138-146, 2004.

MACHADO, J. M.; BORBINHA, J. MITRA: Uma Solução para Serviços de Pesquisa em Intranets. In: XML: Aplicações e Tecnologias Associadas (XATA), 2007. Lisboa. Actas... Lisboa, 2007. Disponível em: <http://xata.fe.up.pt/2007/papers/10.php>. Acesso em: 16 jul. 2007 
MARCUM, D. B. The Future of Cataloging. 2005. Disponível em: $<$ http://www.loc.gov/library/reports/CatalogingSpeech.pdf $>$. Acesso em: 16 jul. 2007.

McCALLUM, S. H. Metadata, Protocol, and Identifier Activities: Library of Congress IFLA/CDNL Alliance for Bibliographic Standards Report, 2004, Buenos Aires. In: WORLD LIBRARY AND INFORMATION CONGRESS: 70th IFLA General Conference and Council, 2004. Proceedings..., Buenos Aires, 2004.2 Disponível em: $<$ http://www.ifla.org.sg/IV/ifla70/papers/024e-McCallum.pdf >. Acesso em: 13 jul. 2007.

ROSETTO, M. Uso do Protocolo Z39.50 para recuperação de informação em redes eletrônicas. Ciência da Informação, v. 26, n.2, p. 136-139, 1997.

SARACEVIC, T. Ciência da informação: origem, evolução e relações. Perspectivas em Ciência da Informação, Belo Horizonte, v. 1, n. 1, p .4162, jan./jun. 1996.

SIQUEIRA, M. A. XML na ciência da informação: uma análise do MARC21. 2003. 134 f. Dissertação (Mestrado em Ciência da Informação) Faculdade de Filosofia e Ciências, Universidade Estadual Paulista, Marília, 2003.

TIOBE Software. TIOBE programming community index for October 2007. 2007. Disponível em: <http://www.tiobe.com/tpci.htm>. Acesso em: 22 out. 2007.

WORLD WIDE WEB CONSORTIUM (W3C). SOAP version 1.2: part 1: messaging framework. 2.ed. 2007. Disponível em: <http://www.w3.org/TR/soap12-part1/>. Acesso em: 10 Ago. 2007.

\section{Anexo A - Servidores SRW/SRU conhecidos}

Disponível em: http://www.loc.gov/standards/sru/servers.html ${ }^{5}$.

BIOME (Internet Resources in the Health and Life Sciences) http://tweed.lib.ed.ac.uk:8080/elf/search/biome?operation=explain\&versi on $=1.1$

COPAC

Database

http://tweed.lib.ed.ac.uk:8080/elf/search/copac?operation=explain\&versi on $=1.1$

Cheshire3

http://srw.cheshire3.org/services/15r

Sample

Databases

http://srw.cheshire3.org/services/spy

http://srw.cheshire3.org/services/syrinnia

${ }^{5}$ Acesso em: 28 abr. 2008. 
EEVL (Internet Guide to Engineering, Mathematics, and Computing) http://tweed.lib.ed.ac.uk:8080/elf/search/eevl?operation=explain\&version $=1.1$

Index Data -- $\quad$ Open Content

Description

SRU

connection information

Example

(Wikipedia

Database):

http://opencontent.indexdata.com/wikipedia

Koninklijke Bibliotheek / The European Library http://krait.kb.nl/cgi-zoek/sru.pl?operation=explain\&version=1.1

Library of Congress Online Catalog http://z3950.loc.gov: $7090 /$ voyager?operation=explain\&version $=1.1$

National Library of Scotland http://tweed.lib.ed.ac.uk:8080/elf/search/nls?operation=explain\&version $=1.1$

OAI Registry at University of Illinois Library at Urbana-Champaign http://gita.grainger. uiuc.edu/registry/sru/sru.asp?operation=explain\&vers ion $=1.1$

OCLC

GSAFD

Database

http://alcme.oclc.org/srw/search/GSAFD?operation=explain\&version=1.1

OCLC PICA SRU Test Database

http://greta.pica.nl:1080/sru/?operation=explain\&version=1.1

OCLC

SOAR

Database

http://alcme.oclc.org/srw/search/SOAR?operation=explain\&version=1.1

Open

University

http://tweed.lib.ed.ac.uk:8080/elf/search/open?operation=explain\&versio

$\underline{\mathrm{n}}=1.1$

Oxford

University

http://tweed.lib.ed.ac.uk:8080/elf/search/oxford?operation=explain\&versi on $=1.1$

Resource Discovery Network ResourceFinder http://www.rdn.ac.uk:8080/xxdefault/?operation=explain\&version=1.1

Social Science Information Gateway

http://tweed.lib.ed.ac.uk:8080/elf/search/sosig?operation=explain\&versio $\underline{\mathrm{n}=1.1}$

University

of

Edinburgh

http://tweed.lib.ed.ac.uk:8080/elf/search/edinburgh?operation=explain\&v ersion $=1.1$

University

of

Glasgow

http://tweed.lib.ed.ac.uk:8080/elf/search/glasgow?operation=explain\&ver $\underline{\text { sion }=1.1}$ 
University

of

Southampton

http://tweed.lib.ed.ac.uk:8080/elf/search/southampton?operation=explai n\&version $=1.1$

University

of

Toronto

http://ibridge.library.utoronto.ca :2200/unicorn?operation=explain\&versio $\underline{n=1.1}$ 